\title{
Rare tumors in pediatrics. First report in Argentina
}

\author{
Alejandra Casanovas, M.D. ${ }^{a}$, Marianela Viso, M.D. ${ }^{b}$, Guido Felizzia, M.D. ${ }^{b}$, \\ Adriana Rose, M.D. ${ }^{b}$, Celeste Mattone, M.D. , Natalia Gazek, M.D. , Bettina Cervini, M.D. , \\ Carlos Giuseppucci, M.D. ${ }^{e}$, Mariana Álvarez, M.D. ${ }^{f}$ and Walter Cacciavillano, M.D. ${ }^{b}$
}

a. Department of Oncology, Hospital General de Niños Pedro de Elizalde.

b. Department of Oncology, Hospital de Pediatría "Prof. Dr. Juan P. Garrahan".

c. Department of Endocrinology, CONICET fellow, Hospital de Pediatría "Prof. Dr. Juan P. Garrahan".

d. Department of Dermatology, Hospital de Pediatría "Prof. Dr. Juan P. Garrahan".

e. Department of Surgery, Hospital de Pediatría "Prof. Dr. Juan P. Garrahan."

f. Department of Pathology, Hospital de Pediatría “Prof. Dr. Juan P. Garrahan".

Autonomous City of Buenos Aires, Argentina.

E-mail address:

Alejandra Casanovas, M.D.:

alito1409@gmail.com

Funding:

This study was conducted in the context of a grant on Pediatric Oncology awarded by the National Argentine Cancer Institute.

Conflict of interests:

None.

Received: 12-11-2020

Accepted: 5-28-2021

\section{ABSTRACT}

Introduction. Collaborative clinical trials have enlarged the knowledge base about pediatric tumors; however, this is not the case for rare tumors (RT).

Objective. To describe the prevalence, clinical characteristics, and course of RT in pediatric patients diagnosed at Hospital Garrahan.

Material and methods. Descriptive, retrospective study of patients aged 0-18 years diagnosed with a RT and admitted between January 2007 and December 2017.

Results. Out of 1657 patients diagnosed with solid tumors, 164 (9.9\%) were RT; $71.95 \%$ (118) of patients were younger than 14 years and $81.7 \%$ (130) were males. In order of frequency, RT were thyroid carcinoma (60), adrenal carcinoma (14), lung tumors (14), melanoma (13), salivary gland cancer (11), gastrointestinal tumors (8), non-germ cell gonadal tumors (7), pancreatic tumors (7), renal carcinomas (6), nasopharyngeal carcinomas (5), pheochromocytoma and paraganglioma (5), and thymic carcinoma in 1 patient. Treatment depended on tumor type and stage. The median follow-up was 34.9 months (range: 1-128.5 months); 133 patients $(78.7 \%$ ) are alive and only 10 patients $(6 \%)$ were lost-to-follow-up. Conclusion. The prevalence of RT was $9.9 \%$. Twenty-seven percent occurred in adolescents. The most frequent tumors included thyroid carcinoma, adrenal carcinoma, and melanoma. Treatment and course varied based on tumor histology. Predisposing molecular alterations were found in $5.3 \%$ of patients; $3.5 \%$ had a history of cancer.

Key words: neoplasms, rare diseases, child, adolescent.

http: / / dx.doi.org/10.5546/ aap.2021.eng.401

To cite: Casanovas A, Viso M, Felizzia G, Rose A, et al. Rare tumors in pediatrics. First report in Argentina. Arch Argent Pediatr 2021;119(6):401-407.

\section{INTRODUCTION}

Pediatric cancer is uncommon. Its incidence has been estimated at $1 / 7000$ children younger than 15 years, with over 12000 new cases per year in patients younger than 20 years in the United States. ${ }^{1}$ The average incidence in Argentina is 1290 cases per year. ${ }^{2}$

RT in pediatrics are defined as "any solid malignancy characterized by an annual incidence below 2/1000 000 and not considered in any research study". ${ }^{3}$ The International Classification of Childhood Cancer (ICCC) ${ }^{4}$ includes $\mathrm{RT}$ in group XI (adrenocortical carcinoma, thyroid carcinoma, nasopharyngeal carcinoma, melanoma, and other unspecified carcinomas), group XII (other specified and unspecified tumors), and group VI (renal tumors), which includes renal carcinomas. Their characteristics include a low prevalence in children younger than 5 years (except for adrenocortical carcinomas), a high prevalence in adults, epithelial etiology and, given the low number of patients, difficulty for their study in research protocols.

The Argentine Hospital Oncopediatric Registry (Registro Oncopediátrico Hospitalario Argentino, ROHA) arranges RT based on their morphology and topography according to the International Classification of Diseases for Oncology, third edition (ICD-O-3) ${ }^{5}$ and the ICCC. ${ }^{4}$ According to this registry, in Argentina, 1290 patients younger than 15 years are diagnosed every year; of these, 367 develop extracranial non-hematopoietic neoplasms. ${ }^{2}$ Fifty percent of patients are seen at Hospital de Pediatría "Prof. Dr. Juan P. Garrahan". 
The objective of this study is to describe the prevalence and characteristics of $\mathrm{RT}$ in pediatric patients diagnosed at Hospital Garrahan, considering the representativeness in the population mentioned above.

\section{MATERIAL AND METHODS}

This was a retrospective and descriptive study of patients aged 0-18 years diagnosed with a RT and admitted between January 2007 and December 2017.

The database included the following: personal data, date of diagnosis (date of pathology report), pathology report, presence of predisposing syndrome or condition, molecular tests performed, treatment, follow-up, and course.

This study was approved by the hospital's Ethics Committee and was conducted respecting and safeguarding patient confidentiality because personal data are not mentioned here.

\section{RESULTS}

In the study period, 4100 patients diagnosed with cancer were admitted to the hospital. A total of 1657 admitted patients were diagnosed with solid tumors. Of them, 164 (9.9\%) had RT (with an average of 16 new patients/year). Table 1 describes tumor distribution by age group and sex.

TABLE 1. Distribution of tumors by age and sex

\begin{tabular}{|c|c|c|c|c|c|}
\hline Tumor & $\mathbf{N}(\%)$ & Median age (years) & $0-14$ years & $15-18$ years & $\operatorname{Sex}(M / F)$ \\
\hline Thyroid carcinoma & $60(36.5)$ & $\begin{array}{c}12.6 \\
(3.57-17.84)\end{array}$ & 45 & 15 & $29 / 31$ \\
\hline Adrenal carcinoma & $14(8.5)$ & $\begin{array}{c}2.8 \\
(0.54-15.7)\end{array}$ & 13 & 1 & $7 / 7$ \\
\hline \multicolumn{6}{|l|}{ Lung tumors: } \\
\hline Pleuropulmonary blastoma & $4(2.4)$ & 2.8 & 4 & 0 & $1 / 3$ \\
\hline Carcinomas & $3(1.8)$ & 16 & 0 & 3 & $0 / 3$ \\
\hline Myofibroblastic tumors & $7(4.2)$ & 5.6 & 5 & 2 & $4 / 3$ \\
\hline Melanoma & $13(7.9)$ & $\begin{array}{c}6.2 \\
(1.56-15.26)\end{array}$ & 11 & 2 & $6 / 7$ \\
\hline Carcinoid tumors & $12(7.3)$ & $\begin{array}{c}12.17 \\
(7.4-16.5)\end{array}$ & 10 & 3 & $7 / 5$ \\
\hline Salivary gland cancer & $11(6.7)$ & 13.3 & 8 & 3 & $4 / 7$ \\
\hline Gastrointestinal tumors: & & $(1.5-14.8)$ & & & \\
\hline Colon cancer & $4(2.4)$ & 15.1 & 0 & 4 & $2 / 2$ \\
\hline GIST & $3(1.8)$ & 15.25 & 1 & 2 & $2 / 1$ \\
\hline GANT & $1(0.6)$ & 14.55 & 0 & 1 & $0 / 1$ \\
\hline Non-germ cell gonadal tumors & $7(4.2)$ & $\begin{array}{c}13.7 \\
(0.15-15.5)\end{array}$ & 4 & 3 & $1 / 6$ \\
\hline \multicolumn{6}{|l|}{ Pancreatic tumors: } \\
\hline Frantz's tumor & $6(3.6)$ & 12.7 & 6 & 0 & $0 / 6$ \\
\hline Pancreatoblastoma & $1(0.6)$ & 5.4 & 1 & 0 & $1 / 0$ \\
\hline Renal carcinoma & $6(3.6)$ & $\begin{array}{c}9.1 \\
(5.8-14.3)\end{array}$ & 6 & 0 & $3 / 3$ \\
\hline Nasopharyngeal carcinoma & $5(3)$ & $\begin{array}{c}14.2 \\
(13.3-16.9)\end{array}$ & 2 & 3 & $3 / 2$ \\
\hline Pheochromocytoma or paraganglioma & $5(3)$ & $\begin{array}{c}10.69 \\
(9.04-15.28)\end{array}$ & 3 & 2 & $3 / 2$ \\
\hline Thymic carcinoma & $1(0.6)$ & 11.5 & 1 & 0 & $1 / 0$ \\
\hline Total & 164 & 12.38 & 118 & 46 & $134 / 30$ \\
\hline
\end{tabular}

GIST: gastrointestinal stromal tumor, GANT: gastrointestinal autonomic nerve tumor. 
Table 2 shows the histology of some tumors, whereas Table 3 describes the predisposing conditions of others.

Patients diagnosed with thyroid carcinoma were managed with a thyroidectomy and radioactive iodine at the Department of Endocrinology. With a median follow-up of 38.4 months (range: $1-128$ months), no patient developed events. Four patients were lost-tofollow-up.

Patients diagnosed with adrenal carcinoma were classified according to the Children's Oncology Group (COG) into stage I (2 patients), stage II (2 patients), stage III (5 patients), and stage IV (5 patients). Patients who had stage I and II tumors were treated with surgery, whereas 4 patients with stage III tumors and 3 with stage IV tumors received chemotherapy. After a median follow-up of 24.6 months (range: 1-66 months), stage I and II patients were alive and disease free. Three patients (stage IV) died: 1 one month after diagnosis, without treatment; the other 2 due to disease progression 6 and 30 months after diagnosis. The patients who received adjuvant treatment did not receive mitotane because this drug is not available in Argentina.

Among the 14 patients who had a lung tumor, 4 were diagnosed with pleuropulmonary blastoma (2 with type I and 2 with type II). The latter received adjuvant treatment: 1 chemotherapy and 1 chemotherapy and radiotherapy; both died due to disease progression 4 and 6 months after diagnosis, respectively. Surgical resection was performed in patients with type I blastoma and were disease free after a median follow-up of 24 months (17 and 31 months, respectively). One patient developed cystic nephroma 2 years after the initial diagnosis.

An inflammatory myofibroblastic tumor was diagnosed in 7 patients; all were treated with surgical resection. Two patients developed local relapse, which was treated with surgery for a new remission; 1 patient died 3 months after diagnosis due to disease progression; 2 patients were lostto-follow-up; and 4 (including the 2 patients who relapsed) are still alive and disease free more than 5 years after diagnosis. Lastly, 3 patients were diagnosed with carcinoma (epidermoid, myoepithelial, and adenoid cystic). The patient with myoepithelial carcinoma underwent surgery and is receiving follow-up by this hospital; the other 2 patients were referred to a different facility. The patient with epidermoid carcinoma received adjuvant chemotherapy and died due to disease progression 15 months after diagnosis. The other 2 patients are alive and disease free 25 and 63 months after diagnosis, respectively.

Out of the 14 patients diagnosed with melanoma, 8 are alive and disease free, with a median follow-up of 26 months (range: 2-89 months). Four patients died due to disease progression 2, 4, 19, and 26 months after diagnosis, respectively. Two patients were lostto-follow-up.

The 12 patients with carcinoid tumors ( 5 in the lung and 7 in the vermiform appendix) were treated with surgery only. They are currently alive and disease free after a median follow-up of 35 months (range: 3-71 months).

Among the 11 patients diagnosed with salivary gland cancer, only the patient with poorly differentiated carcinoma developed metastasis and was the only one who received adjuvant treatment (chemotherapy and radiotherapy); this patient died due to disease progression 8 months after diagnosis. One patient with mucoepidermoid carcinoma relapsed 55 months after diagnosis and achieved a new remission with surgery. Nine patients are alive and disease free after a median follow-up of 29 months (range: 2-79 months). One patient was lost-to-follow-up.

The 4 patients who had colon cancer had metastasis at the time of diagnosis and were treated with chemotherapy; 1 of them was referred to another facility. All these patients died due to disease progression, with a median survival after diagnosis of 6.7 months (range: 5-11 months).

The patients diagnosed with gastrointestinal stromal tumor (GIST) (3 patients) and gastrointestinal autonomic nerve tumor (GANT) (1 patient) were treated only with surgery. One patient diagnosed with GIST developed regional relapse 24 months after diagnosis, received imatinib for 2 years, remained stable and is alive 79 months after diagnosis. The other 3 patients are alive and disease free, with a follow-up between 5 and 20 months.

The 7 patients who had non-germ cell gonadal tumors were classified after surgery into stage I (3 patients) and stage II (4 patients) as per the Intergroup Rhabdomyosarcoma Studies (IRS) classification following surgery. Only ovarian small cell carcinoma showed metastasis. Treatment was administered to 5 patients: 4 received chemotherapy ( 1 relapsed) and 1 (small cell carcinoma) received chemotherapy and radiotherapy and died 3 months after diagnosis 
TABLE 2. Tumors by histologic subtype

\begin{tabular}{ll}
\hline Tumor (n patients) & Histologic subtype (n patients) \\
\hline Thyroid carcinoma (60) & Papillary (50) \\
Follicular (7) & Medullary (3) \\
Salivary gland cancer (11) & Mucoepidermoid (7) \\
& Acinic cell (2) \\
& Adenoid cystic (1) \\
& Poorly differentiated (1) \\
Non-germ cell gonadal tumors (7) & Juvenile granulosa (3) \\
& Sertoli-Leydig (2) \\
Sclerosing stromal (1) \\
Ovarian small cell carcinoma (1) \\
Renal carcinoma (6) & Clear cell (4) Papillary (1) \\
& Chromophobe subtype (1) \\
Pheochromocytoma/paraganglioma (5) & Pheochromocytoma (2) \\
& Paraganglioma (2) \\
\end{tabular}

TABLE 3. Genetic-molecular alterations predisposing to cancer observed in this study

\begin{tabular}{|c|c|}
\hline Tumor (total patients) & Alteration and number of patients \\
\hline Thyroid carcinoma (60) & $\begin{array}{l}\text { Previous disease ( } 8 \text { patients): } 6 \text { developed thyroid carcinoma as second } \\
\text { neoplasm ( } 2 \text { with Hodgkin lymphoma treated with radiotherapy in the } \\
\text { neck area, } 1 \text { with a history of Sertoli-Leydig cell tumor with mutation in } \\
\text { the DICER-1 gene, } 1 \text { with nasopharyngeal rhabdomyosarcoma treated } \\
\text { with radiotherapy, } 1 \text { diagnosed with acute lymphoblastic leukemia and } \\
\text { hypopituitarism who developed testicular relapse treated with radiotherapy, } \\
\text { and } 1 \text { with hepatocellular carcinoma associated with type I glycogen storage } \\
\text { disease who underwent liver transplantation). One patient } \\
\text { had a history of pituitary adenoma and another, autoimmune } \\
\text { nodular goiter. }\end{array}$ \\
\hline
\end{tabular}

Adrenal carcinoma (14)

Pheochromocytoma

Nasopharyngeal carcinoma (5)

Non-germ cell gonadal tumors (7)

Pleuropulmonary blastoma (4)
A mutation in the $p 53$ gene was studied in 6 patients and detected in 3 . One of these patients had been diagnosed with

Li-Fraumeni syndrome, 1 had the most common mutation observed in patients with this type of tumor in the south of Brazil (p.R337H), and 1 had a sporadic mutation.

VHL mutation (1 patient).

Three patients were tested for Epstein-Barr virus DNA using a PCR and it was detected in all 3.

Two patients were positive for the DICER-1 gene mutation (both corresponded to Sertoli-Leydig cell tumors).

Two patients were tested for the DICER-1 gene mutation (both type I), both were negative.

DNA: deoxyribonucleic acid. 
due to disease progression. The remaining patients are alive and disease free after a median follow-up of 18 months (range: 8-119 months).

Six patients developed solid pseudopapillary tumors of the pancreas (Frantz's tumor) and were managed with surgery only. One patient who had localized pancreatoblastoma was treated with chemotherapy (cisplatin and doxorubicin) and ended the treatment in full remission. This patient is alive and disease free 7 months after diagnosis.

Out of the 6 patients diagnosed with renal carcinoma, 5 underwent nephrectomy. Nodal involvement was observed in 3 patients, none had metastasis at the time of diagnosis. Two patients received chemotherapy and radiotherapy; 1 patient underwent radiotherapy; and the rest, only surgical treatment. The patient who had a biopsy performed did not receive any treatment and died 21 months after diagnosis. One patient developed metastasis 5 months after diagnosis and died 6 months later. The other 4 patients are alive and disease free after a median follow-up of 52 months (range: 18-86 months).

Among the patients diagnosed with nasopharyngeal carcinoma, 3 had metastasis at the time of diagnosis. All underwent treatment (chemotherapy and radiotherapy in 4 and radiotherapy alone in 1). Two patients developed metastasis; 1 died 33 months after diagnosis. The remaining patients are alive and disease free after a median follow-up of 60 months (range: 26-105 months).

The patients diagnosed with pheochromocytoma or paraganglioma were treated with surgery. One of them had metastasis at the time of diagnosis and died 50 months after diagnosis. Three patients are disease free after a median follow-up of 60 months (range: 2253 months); and 1 was lost-to-follow-up.

The patient diagnosed with thymic carcinoma developed metastasis. The resected specimen margins were compromised. After surgery, the patient received chemotherapy and radiotherapy and died 7 months after diagnosis due to disease progression.

No patient had breast cancer.

\section{DISCUSSION}

This is the first report on RT in Argentina. Although it was conducted at a single facility, Hospital Garrahan caters for $50 \%$ of patients with solid tumors at a national level. The concept of representativeness is reinforced when comparing the total of patients diagnosed with a RT and recorded in the $\mathrm{ROHA}^{2}$ in the 2000-2013 period, with an average of 30 patients per year, to this study conducted over a 10-year period, with an average of 16 patients per year.

Since this was a retrospective study, with patients treated at different hospital departments, the number of recorded patients may be lower than the actual number of patients. Thus, this is considered one of the main weaknesses of the study, similar to what has been observed in the first report of the Rare Tumors in Pediatric Age (TREP, for its Italian acronym) project, ${ }^{3}$ who made a retrospective description for the 19821998 period and found a frequency of 15 new patients per year; with a prospective study, this figure increases to 49 patients per year (between January 2000 and December 2005). It is also worth noting that, whereas the Italian group and the COG report that RT correspond to 8-10

$\%$ of all tumors diagnosed in pediatric patients, ${ }^{3,6}$ in our cohort, out of 4100 patients admitted with cancer, 164 had a RT (4\%).

According to the Surveillance, Epidemiology and End Results (SEER) Program, ${ }^{7} 75 \%$ of RT occur in adolescents and young adults. In our cohort, patients older than 15 years account $27 \%$, similar to what has been reported by the Italian group ( $22 \%$ of patients were between 15 and 17 years old ${ }^{3}$ ). Most likely, this is because patients diagnosed with RT during adolescence are managed at adult care facilities.

In our series, the most frequent tumors included thyroid carcinoma, adrenal carcinoma, and melanoma. When grouped by age, the same frequency order is observed among children (0-14 years old), whereas among adolescents (15-18 years old), thyroid carcinoma remains as the leading type, followed by colon cancer in the second place. The rest showed a similar distribution. It is worth noting that patients diagnosed with thyroid carcinoma receive follow-up and treatment only at the hospital's Department of Endocrinology, except for those who had a previous neoplasm. Something similar occurs with patients diagnosed with melanoma, who are seen at the Department of Dermatology and rarely attend the Department of Oncology. This is a limitation when it comes to recording patients, also observed by other international groups. . $^{3-6-8}$

A characteristic of some of these RT is that they show specific molecular alterations. Knowledge of their presence is critical for an adequate genetic counseling and a chance to study family members of the index patient for an eventual early diagnosis. 
This study found that only $43 \%$ of patients diagnosed with adrenal carcinoma are tested for a mutation in the $p 53$ gene, which had an alteration in $50 \%$ of cases. It would be important to know p53 gene status because it is known that there is a higher incidence of this type of tumor in the south of Brazil due to a specific mutation, ${ }^{9-10}$ and that the hospital receives a large number of patients from provinces bordering Brazil.

Von Hippel-Lindau syndrome is a familial disease predisposing to cancer, associated with several benign and malignant tumors, mostly in the retina and cerebellum, and spinal cord hemangioblastoma, renal cell carcinoma and, to a lower extent, pheochromocytoma. ${ }^{11}$

This is due to a mutation in the VHL gene, located in chromosome 3p25.3; it is an autosomal dominant inherited syndrome and its detection in 1 of the patients who had pheochromocytoma may be useful not only for genetic counseling purposes, but also to perform periodic controls to rule out the other frequent tumors in this syndrome.

Another mutation worth studying is common to 2 diseases, the one in the DICER-1 gene (14q32.13). It is usually associated with pleuropulmonary blastomas, ovarian sex cordstromal tumors, and cystic nephromas, among others. ${ }^{12}$ Among the patients in this series who had a diagnosis of pleuropulmonary blastoma, only those with type I tumors were tested: one of them developed cystic nephroma; both were negative for the mutation. In relation to patients diagnosed with non-germ cell gonadal tumors, this mutation was found in both patients diagnosed with Sertoli-Leydig cell tumor, described in the bibliography as the most common histology for this mutation.

The collection of data in relation to molecular alterations regarding the patients described here indicates that not all of them were tested, mostly because the test was not available at the time of diagnosis.

A relevant piece of information found in this study is that $10 \%$ of patients diagnosed with thyroid carcinoma had a history of previous cancer. Four of them had been treated with radiotherapy (2 specifically in the neck area), which increases the risk for thyroid carcinoma by $17 \%$ because the thyroid is highly susceptible to the carcinogenic effects of radiation. ${ }^{13}$ One patient received immunosuppressive therapy for a transplant and another showed a mutation in the DICER-1 gene. This reinforces the importance of follow-up and control after treatment in cancer patients.

In relation to the follow-up of patients diagnosed with RT, it is worth mentioning that only $6 \%$ were lost-to-follow-up. Knowing this information will allow to implement new recording strategies and develop diagnosis, treatment, and follow-up recommendations in an interdisciplinary manner. Data collection from multiple sites and at a national level is the challenge for the next step.

\section{CONCLUSION}

Among solid tumors, the prevalence of RT was $9.9 \%$. Twenty-seven percent of patients were adolescents. The most frequent tumors included thyroid carcinoma, adrenal carcinoma, and melanoma. Treatment and course varied based on tumor histology. Predisposing molecular alterations were found in $5.3 \%$ of patients; $3.5 \%$ had a history of cancer.

\section{REFERENCES}

1. Gurney JG, Bondy ML. Epidemiology of childhood cancer. In: Pizzo PA, Poplack DG (eds). Principles and practice of pediatric oncology. $5^{\text {th }}$ ed. Philadelphia, PA: Lippincott Williams \& Wilkins; 2006.Pages.1-13.

2. Moreno F, Chaplin MA. Registro oncopediátrico hospitalario argentino. $6^{\mathrm{a}}$ ed. Ciudad Autónoma de Buenos Aires: Instituto Nacional del Cáncer; 2018.

3. Ferrari A, Bisogno G, De Salvo GL, Indolfi P, et al. The challenge of very rare tumours in childhood: The Italian TREP project. Eur J Cancer. 2007; 43(4):654-9.

4. International Classification of Childhood Cancer. Subgroup XI, of the SEER database 2003-2007. [Accessed on: June $1^{\text {st }}$, 2021]. Available at: http: / / seer.cancer.gov/csr /1975_2007/results_merged / sect_29_childhood_cancer_ iccc.pdf

5. Fritz A, Perci C, Jack A, Shanmugaratnam K, et al (eds). ICD-O International classification of diseases for oncology. $3^{\text {rd }}$ ed. Geneva: World Health Organization; 2000.

6. Pappo AS, Krailo M, Chen Z, Rodríguez-Galindo C, et al. Infrequent tumor initiative of the Children's Oncology Group: Initial lessons learned and their impact on future plans. J Clin Oncol. 2010; 28(33):5011-6.

7. Ries L, Smith M, Gurney J, Linet M, et al (eds). Cancer Incidence and Survival among Children and Adolescents: United States SEER Program 1975-1995. Bethesda, MD: National Cancer Institute, SEER program NIH Pub No. 99-4649; 1999.

8. Villarroel M, Chantada G. Tumores raros en niños y adolescentes. Rev Med Clin Condes. 2015; 26(4):495-502.

9. Rodríguez-Galindo C, Pappo AS, Krailo MD, Pashankar $\mathrm{F}$, et al. Treatment of childhood adrenocortical carcinoma (ACC) with surgery plus retroperitoneal lymph node dissection (RPLND) and multiagent chemotherapy: results of the Children's Oncology Group ARAR0332 protocol. J Clin Oncol. 2016; 34(Suppl 15):10515.

10. Ribeiro RC, Pinto E, Zambetti G, Rodríguez-Galindo C. The International Pediatric Adrenocortical Tumor Registry initiative: Contributions to clinical, biological, and treatment advances in pediatric adrenocortical tumors. Mol Cell 
Endocrinol. 2012; 351(1):37-43.

11. Varshney N, Kebede AA, Owusu-Dapaah H, Lather J, et al. A review of von Hippel-Lindau syndrome. J Kidney Cancer VHL. 2017; 4(3): 20-9.

12. Schultz KA, Stewart DR, Kamihara J, Bauer AJ, et al.
DICER1-Tumor predisposition. In: Adam M, Ardinger HH, Pagon RA, Wallace SE, et al (eds). GeneReviews. Seattle, WA: University of Washington; 2014.

13. Waguespack SG. Thyroid Sequelae of Pediatric Cancer Therapy. Horm Res Paediatr. 2018; 91(2):104-17. 